\title{
An upper bound on the number of errors corrected by a convolutional code
}

\author{
Justesen, Jørn
}

Published in:

IEEE International Symposium on Information Theory, 2000. Proceedings.

Link to article, DOI:

10.1109/ISIT.2000.866587

Publication date:

2000

Document Version

Publisher's PDF, also known as Version of record

Link back to DTU Orbit

Citation (APA):

Justesen, J. (2000). An upper bound on the number of errors corrected by a convolutional code. In IEEE International Symposium on Information Theory, 2000. Proceedings. IEEE.

https://doi.org/10.1109/ISIT.2000.866587

\section{General rights}

Copyright and moral rights for the publications made accessible in the public portal are retained by the authors and/or other copyright owners and it is a condition of accessing publications that users recognise and abide by the legal requirements associated with these rights.

- Users may download and print one copy of any publication from the public portal for the purpose of private study or research.

- You may not further distribute the material or use it for any profit-making activity or commercial gain

- You may freely distribute the URL identifying the publication in the public portal

If you believe that this document breaches copyright please contact us providing details, and we will remove access to the work immediately and investigate your claim 


\title{
An Upper Bound on the Number of Errors Corrected by a Convolutional code
}

\author{
Jørn Justesen \\ Department of Telecommunication \\ Technical University of Denmark, Building 371 \\ DK-2800 Lyngby, Denmark \\ e-mail: jju@tele.dtu.dk
}

\begin{abstract}
The number of errors that a convolutional codes can correct in a segment of the encoded sequence is upper bounded by the number of distrinct syndrome sequences of the relevant length.
\end{abstract}

\section{INTRODUCTION}

We shall analyse the error correcting power of a convolutional code by relating the number of correctable errors to the available syndromes. The results are related to the bound in [1], but we take a more direct approach. Syndromes for convolutional codes have not received much attention since the structural results appeared in [2]. The main difficulty compared to block codes, is that different sequence lengths have to be considered. Even though a Hamming type upper bound usually cannot be reached, it is an important estimate of the number of errors that can be corrected with high probability by a typical code.

\section{CORRECTABLE ERRORS FOR SHORT SEQUENCES}

In [3] a general method for relating bounds for block codes to convolutional codes was introduced. Thus an upper bound on the number of errors that can be corrected independent of their location, $\mathrm{t}_{0}$, may be derived from the Hamming bound for block codes. However, a direct analysis of errors and syndromes in convolutional codes gives a tighter bound, since some error patterns give rize to short syndromes.

Theorem 1: If a binary $(\mathrm{n}, \mathrm{k})$ convolutional code with encoder memory $\mathrm{M}$ (blocks) and syndrome former memory $\mathrm{M}$ ' corrects all combinations of $t_{0}$ errors, the inequality

$$
\sum\left(\begin{array}{c}
n s \\
j
\end{array}\right) c(j) \leq 2^{(n-k)\left(s+M^{\prime}\right)}
$$

is satisfied for any $s>M$ and $j \leq t_{0}$. Here $c(j)$ is the number of truncated codewords of weight $j$.

The bound will be applied to examples of short high rate codes, and we shall demonstrate how the factor $\mathrm{c}(\mathrm{j})$ makes the bound sharper than the translated Hamming bound. It is essential to the performance of convolutional codes that the number of correctable errors increases with the length of the sequence. Thus we are interested in the number of errors, $t_{j}$, that can be corrected in a sequence of length $j$ blocks, provided that no more than $t_{0}$ errors occur in a sequence of length $j-1$. This approach may be extended to yield a description of distributions of correctable errors in short sequences.

\section{A VARIABLE LENGTH DESCRIPTION OF ERRORS}

An obvious question about a convolutional code is, how often can a burst of $t_{0}$ errors be corrected? Our first ajpproach above does not give a convenient answer to questions of this type, since the syndromes are simply assumed to be zero outside the window under consideration. Thus we seek a rule for segmenting the error pattern into finite strings in such a way that any concatenation of correctable strings form a correctable error sequence. This gives a variable length description of the correctable error patterns which may be related to a segmentation of the syndrome sequence. The segments may be mapped on the leaves of a tree where the branches are labeled by the syndrome bits.

\section{AN UPPER BOUND BY THE KRAFT INEQUALITY}

We may obtain a Hamming type upper bound by relating the error sequence and the syndrome sequence through a version of Kraft's inequality:

Theorem 2: For a tree of correctable error patterns, the number of paths of length $L$ (blocks) is $A(L)$. Then the number of check symbols per block, $r$, must satisfy

$$
\sum A(L) 2^{r L} \leq 1
$$

This version of the upper bound indicates that for short codes there is a trade-off between a high value of $t_{0}$ and a rapid increase in the number of correctable errors with the length of the sequence. Clearly for long codes, the fraction of errors is given by the saymptotic Hamming bound.

\section{RELATION TO THE BOUND BY FINITE STATE ALGORITHMS}

While the bound of Theorem 2 gives a convenient way of testing partial descriptions of error patterns, the variable length description usually leads to an infinite tree. Thus a complete weight specification is naturally described by a finite state algorithm, and we arrive at the upper bound discussed in [1].

\section{REFERENCES.}

[1] J. Justesen, "Bounded distance decoding of unit memory codes," IEEE Trans. Info. Th., vol. IT-39, September 1993, pp. 16161627.

[2] G. David Forney, Jr., "Structural analysis of convolutional codes via dual codes," IEEE Trans. Info. Th., vol. IT-19, July 1973, pp. 512-518.

[3] G. David Forney, Jr., "Convolutional codes II: Maximum likelihood decoding," Information and Control, vol. 25, July, 1974, pp. 222-266. 